\title{
RELACIÓN ENTRE LAS TENDENCIAS TEMPORALES DE PRODUCCIÓN Y TRANSPORTE DE SEDIMENTOS Y LAS CONDICIONES CLIMÁTICAS EN UNA PEQUEÑA CUENCA DE MONTAÑA MEDITERRÁNEA (VALLCEBRE, PIRINEOS ORIENTALES)
}

\author{
D. REGÜÉS \\ J.C. BALASCH \\ X. CASTELLTORT \\ M. SOLER \\ F. GALLART
}

Institut de Ciènces de la Terra Jaume Almera (CSIC), c/ Solè i Sabarís s/n. Barcelona 08025

C. electrónico: fgallart@ija.csic.es

\begin{abstract}
RESUMEN: La cuenca de Vallcebre (cabecera del río Llobregat) muestra una importante variedad de usos del suelo y ambientes geomorfológicos: bosques, pastos, terrazas cultivadas y abandonadas, y cárcavas. Esto ba llevado a la selección de tres cuenca integradas (Cal Rodó, Ca L'Isard y Can Vila) equipadas para cuantificar la producción de escorrentía y sedimento en distintas condiciones. Además se dispuso una red de pluviómetros y dos estaciones meteorológicas en la cuenca. Las zonas acarcavadas, únicamente presentes en las cuencas de Cal Rodó y Ca L'Isard, son relativamente pequeñas pero muy activas, por dos motivos: una precipitación abundante (924 $\mathrm{mm}$ año ${ }^{-1}$ ) y unas elevadas tasas de meteorización en invierno. Entre septiembre de 1994 y diciembre de 1999 fueron registradas unas $350 \mathrm{creci}$ das en las estaciones de Cal Rodó y Ca L'Isard. En la estación de Can Vila solamente fueron registradas una pocas decenas de crecidas, ya que no estuvo completamente equipada basta junio de 1998, en este caso la ausencia de cárcavas quedó evidenciada por la baja producción de sedimentos.

Con el fin de estudiar las tendencias temporales del transporte de sedimentos se realizaron análisis de correlaciones, en las que se relacionaron los parámetros sedimentológicos con los bidrológicos, considerando las variables a diferentes escalas temporales (evento individual, mes, estación $y$ año). Los resultados muestran que las tasas de transporte dependen de las condiciones climáticas estacionales, directamente de la distribución de las precipitaciones e indirectamente de los regímenes anuales de humedad y temperatura, que afectan a la dinámica de meteorización en las cárcavas. La principal conclusión relacionada con la escala temporal sugiere que los resultados y su interpretación dependen de la estación o el año considerado. La tasa media de erosión puede cambiar por un evento individual, porque aproximadamente el $90 \%$ del transporte se produce en un 2 $\%$ de las crecidas y un $17 \%$ de la precipitación total. Esto significa que, en estas zonas de cárcavas, la distribución y características de las precipitaciones (no la cantidad total) con respecto a la dinámica de meteorización en las cárcavas, son los principales factores que controlan las tasas de erosión y el transporte.
\end{abstract}


ABSTRACT: The Vallcebre catchment (Alobregat river bead-waters) sbows an important variety on land uses and geomorphic environments: forest, pasture, farmed and abandoned agricultural terraces, and badlands. Three nested catchments (Cal Rodó, Ca L'Isard and Can Vila) were equipped to measure bydrological and sedimentological yield from different land conditions. There was also set a raingauge net and two weather stations in the basin. Badland areas, that occur only in the Cal Rodo and Ca l'Isard catchments, are relatively small but very active, because both the abundant precipitation (924 $\mathrm{mm}^{\text {year }}{ }^{1}$ ) and the high weathering rate in winter.

Between September 1994 and December 1999 about 350 events were recorded at both the Cal Rodo and the Ca L'Isards station. At the Can Vila station some few tens of events were recorded, because it was not completely equipped until June 1998, in this case the badlands absence was reflected in a very poor sediment production.

To study the temporal patterns on sediment transport a set of statistical analysis was performed, relating sedimentary parameters with the bydrological ones, at different temporal scales (single event, month, season and year). The results show that transport rates depend on the seasonal climatic conditions, directly by rainfall distribution, and indirectly by moisture and temperature annual regimes, because their great influence on the weathering badland dynamics. The most important conclusion related with the temporal scale suggested that results and their interpretation depend on the season or year taken into account. Mean erosion rates can be changed by a single event, because approximately a $90 \%$ of the sediment yield is produced by only a $2 \%$ of the events and a $17 \%$ of the total precipitation. This means that, in these badland areas, precipitation distribution and characteristics (nor total amount), along with badland weathering dynamics are the main factor controlling erosion rates and transport.

Palabras clave: Usos del suelo, Cuencas experimentales, Cárcavas, Montaña húmeda, Dinámica de cárcavas, Producción de sedimento, Condiciones climáticas, Escala temporal.

Key words: Land uses, Experimental catchment, Badland, Humid mountain, Badland dynamics, Sediment yield, Climate conditions, Temporal scale.

\section{Introducción}

Las cuencas de Vallcebre (cabecera del río Llobregat) han sido el marco de estudios relacionados con procesos relacionados con la producción y el transporte de sedimentos desde 1986. Como consecuencia de ello ha derivado un amplio número de publicaciones: Clotet y Gallart (1986), Balasch et al., (1988), Clotet et al. (1988), Solé et al. (1992), Regüés et al. (1995 y 2000), Regüés y Gallart (1996), Pardini et al. (1995 y 1996), Llorens et al. (1997), Gallart et al. (1998), entre otros, en los que se identifican y, en algunos casos, cuantifican los principales procesos de meteorización, erosión y transporte de sedimento activos en los distintos ambiente localizados en las Cuencas de estudio, que por otro lado, también han sido relacionados con las características de la vegetación mediante los trabajos de Guàrdia y Ninot, (1992) y Guàrdia (1995). Todo ello ha proporcionado una amplia perspectiva relacionada con el ciclo dinámico de los sedimentos, desde las principales áreas 
fuente (cárcavas), hasta su incorporación a los principales canales torrenciales de drenaje, que facilitan la exportación hacia la red fluvial de mayor jerarquía.

Para la adquisición de este importante volumen de información se realizó una planificación experimental a largo plazo, fundamentada en la observación, captación de datos y experimentación a diferentes escalas espaciales y temporales. Lo que implicó un tránsito gradual y racional, desde la monitorización y experimentación en pequeñas parcelas experimentales, donde se centraron los estudios relacionados con procesos de meteorización y erosión, pasando por microcuencas en las que se pudieron evaluar tasas de producción de sedimento, hasta llegar a la escala de cuencas aforadas, que han proporcionado una idea sobre el transporte y exportación del sedimento.

\section{Area de estudio}

La pequeña cuenca de Vallcebre $\left(19.6 \mathrm{~km}^{2}\right)$ está localizada en la cabecera del río Llobregat, en el sector sudoriental de los Pirineos, entre altitudes que van de los 960 a $\operatorname{los} 2245$ m. s. n. m. El clima de la zona ha sido definido como submediterráneo (Martín-Vide, 1985), que se caracteriza por un régimen medio anual de precipitaciones de unos $925 \mathrm{~mm} \mathrm{a}^{-1}$ (con una marcada variabilidad interanual) y una temperatura media anual de unos $9^{\circ} \mathrm{C}$. Sin embargo, la característica más destacable de la climatología está definida por una marcada estacionalidad de los regímenes térmico e hídrico.

Así, han sido identificadas dos estaciones húmedas: primavera y otoño, caracterizadas por una distribución temporal de las precipitaciones generalmente homogénea, y dos estaciones secas: un invierno marcadamente seco, en el que las precipitaciones suelen ser en forma de nieve y un verano que inicialmente suele presentar una etapa de déficit hídrico, acentuada por unas temperaturas muy elevadas, que suele estar seguido de un periodo marcado por frecuentes tormentas convectivas de corta duración y alta intensidad.

Por otro lado, el régimen térmico anual también muestra dos periodos bien definidos. Una estación fría entre octubre y abril, donde las temperaturas pueden descender por debajo de los $-10^{\circ} \mathrm{C}$, con oscilaciones térmicas diarias de $5-15^{\circ} \mathrm{C}$ que causan abundantes ciclos de hielo-deshielo en la superficie del terreno sin vegetación, entre 90 y 110 anuales en vertientes orientadas hacia el $\mathbf{N}$. Una estación cálida, entre mayo y septiembre, con temperaturas máximas en los meses estivales que superan los $30^{\circ} \mathrm{C}$ y oscilaciones térmicas diarias de $10-20^{\circ} \mathrm{C}$; en este caso la superficie del terreno sin vegetación y en vertientes orientadas hacia el $\mathrm{S}$ puede llegar a soportar oscilaciones diarias que superan los $40^{\circ} \mathrm{C}$, cuando las temperaturas en superficie se aproximan a los $60^{\circ} \mathrm{C}$ durante los meses de julio y agosto a causa de una elevada radiación solar (Regüés, 1995).

En cuanto a las características geológicas, la cuenca de Vallcebre está integrada en la estructura definida por la denominada "Cubeta de Vallcebre" (Martínez y Vergés, 1978) y se constituye por una secuencia de tipo regresiva continental en la que se alternan calizas y margas de la facies Garumniense, denominada Fm Tremp (Feist y Colombo, 1983). Así, los márgenes de la cuenca están formados por calizas verti- 
calizadas que constituyen los flancos de una secuencia de plegamientos cónicos, mientras que el núcleo está formado por materiales margosos o arcillosos continentales, caracterizados por presentar un elevado porcentaje de esmectitas y una textura en un $90 \%$ limo-arcillosa.

Este conjunto de características climático-geológicas, unidas a las modificaciones de carácter antrópico, ha dotado la Cuenca de un amplio mosaico de ambientes geomorfológicos (bosques, prados, campos y terrazas abandonadas, superficies estructurales sobre calizas y pequeñas cárcavas) en una reducida extensión de territorio. Esta peculiar distribución de ambientes ha permitido la identificación y selección de un conjunto de cuencas experimentales, que al quedar integradas mediante la red de drenaje han facilitado un estudio individualizado y secuencial de las respuestas hidrológicas y sedimentológicas, proporcionando con ello una comparación entre las citadas situaciones y constituyendo un marco excepcional en cuanto a laboratorio experimental natural.

\section{Estudios antecedentes}

\subsection{Dinámica de las cárcavas en relación con la producción de sedimentos.}

Los estudios específicos sobre la dinámica de las cárcavas se localizaron en una zona de unos 0,06 km² conocida como El Carot (Regüés 1995, Castelltort 1995 y Pardini 1996). Estos trabajos fueron desarrollados mediante un seguimiento de parcelas experimentales abiertas, en el campo, y el complemento de experiencias de simulación, en el laboratorio. Como resultado se observó una marcada tendencia estacional en los procesos de producción (meteorización y erosión) así como una dinámica extremadamente acelerada.

Estas características quedaron evidenciadas en algunos indicadores del estado físico del regolito superficial (densidad aparente y humedad gravimétrica) y su relación con las variaciones estacionales de las condiciones hídricas y térmicas. Esta buena relación quedó demostrada en las variaciones de la densidad aparente (0-5 $\mathrm{cm}$ ), considerada el principal indicador del grado de meteorizazión del regolito superficial, y la humedad gravimétrica $(0-10 \mathrm{~cm})$, mediante dos ecuaciones ajustadas a una función del seno del día juliano (Fig. 1):

$$
\begin{aligned}
& \text { hv }=16-(7.7 \cdot \operatorname{sen}(2 \cdot \pi \cdot(d-110)) / 365) / d a \quad(n: 65, \mathrm{R}: 0.83, \text { conf. } 5 \%) \\
& \mathrm{da}=1.1009-0.2 \cdot\left(\operatorname{sen}\left(2 \cdot \pi \cdot(\mathrm{d}+46.93)^{1.009 \cdot h \mathrm{hv})}\right) / 365 \quad(\mathrm{n}: 65, \mathrm{R}: 0.79, \text { conf. } 5 \%)\right. \\
& \text { hv: humedad volumétrica, da: densidad aparente, d:día juliano }
\end{aligned}
$$

Este comportamiento de tipo armónico es un reflejo de la acción secuencial de los procesos de meteorización y erosión, que mediante su ciclo anual característico definen tres etapas principales:

a) Desde mediados de otoño a invierno predomina la meteorización física del regolito, por la acción del hielo-deshielo (Regüés et al 1995 y 2000). Como resultado 


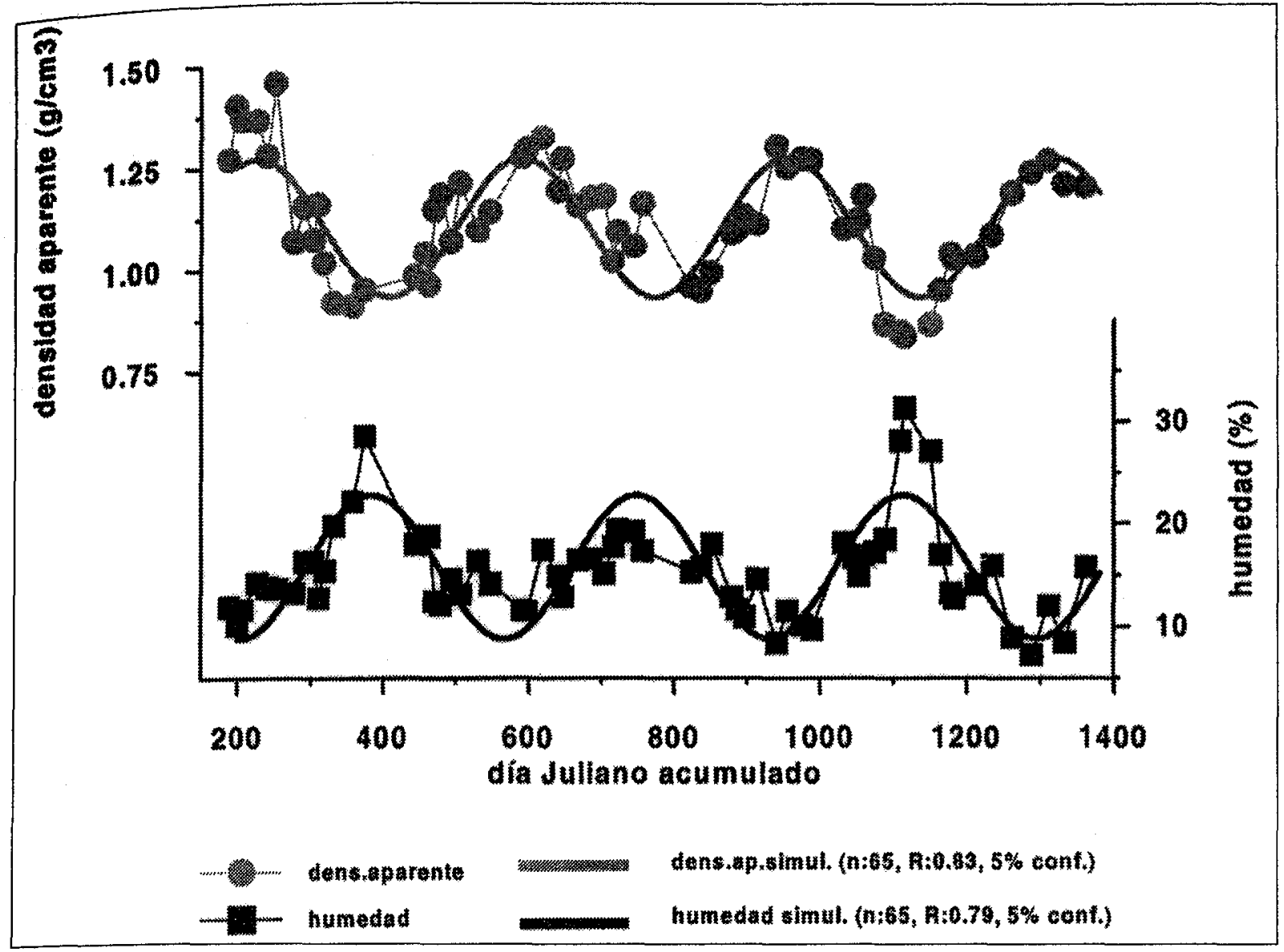

Figura 1. Tendencias temporales de la densidad aparente y la bumedad del regolito superficial en las cárcavas de "El Carot"

se desarrolla un estrato de regolito superficial muy frágil, tipo "popcorn" (Regüés et al., 1994; Regüés, 1995) con una elevada porosidad que favorece la infiltración sobre la generación de escorrentía superficial. La formación del "popcorn" como consecuencia del hielo-deshielo ha sido verificada mediante experimentos de laboratorio (Regüés et al. 1994; Pardini et al. 1996).

b) Desde primavera hasta mediados de verano predominan los procesos de erosión en las laderas (Regüés y Gallart, 1996 y en prensa) asociados a los cambios de las condiciones ambientales de carácter estacional. En estas condiciones se activan los mecanismos de erosión que producen la desintegración y encostramiento del regolito altamente meteorizado o "popcorn", porque el incremento de las temperaturas causa el desecado del regolito, y en esta situación la superficie se muestra altamente susceptible al impacto de las gotas de lluvia o "splash", iniciando la compactación del estrato alterado por un efecto combinado de desagregación y redistribución del particulado (Mc Intyre, 1958; Slattery and Bryan, 1994). Mientras el consiguiente efecto de la humectación-desecación contribuye positivamente al desarrollo de la costra superficial (Regüés, 1995; Pardini et al. 1996). Contemporáneamente una parte del material erosionado es transportado hasta el pié de las vertientes, depositándose junto a los márgenes de los canales principa- 
les en forma de abanicos detríticos, constituyendo un importante "stock" de sedimento disponible (Regüés, 1995; Castelltort, 1995).

c) Con la llegada de la estación más húmeda, entre finales del verano y otoño, la costra superficial facilita un sellado rápido, lo que permite respuestas hidrológicas casi instantáneas. En estas condiciones predomina el transporte, ya que a causa de los elevados coeficientes de escorrentía observados en las cárcavas (Regüés 1995; Regüés y Gallart, 1996 y en prensa; Balasch, 1998) se originan caudales capaces de barrer los abanicos de sedimentación (Castelltort, 1995; Gallart et al., 1998) y limpiar en mayor o menor grado las vertientes de las cárcavas, dejando expuesta la roca a la acción de los procesos meteorizantes. El transporte a larga distancia viene facilitado por los caudales elevados generados en el conjunto de la cuenca durante la estación húmeda (Gallart et al., en prensa)

Estas observaciones sugieren la existencia de un efecto de proporcionalidad inversa entre el estado de meteorización de la roca superficial y la efectividad con que se genera la escorrentía superficial o erosiona el material. Lo que confirma la hipótesis inicialmente formulada por Clotet y otros (1998), que presupone un control de los ritmos de meteorización sobre las tasas de erosión, aunque precisando que el control entre ambos procesos es recíproco (Regüés, 1995), porque sin una eliminación previa del regolito alterado queda limitada la acción penetrativa de los procesos de meteorización.

\subsection{Erosión y transporte en las cárcavas}

Instrumentación. En el estudio de los procesos de transporte del sedimento se ascendió desde la escala de parcelas de simulación de lluvia $\left(0,225 \mathrm{~m}^{2}\right)$ hasta la de parcela de erosión y pequeña cuenca. Para ello fueron seleccionados tres puntos en la red de canales de drenaje de la cuenca de Cal Rodó (sector meridional de la cuenca de Vallcebre) y equipados con sistemas de medición de caudales y sedimentos (Balasch et al., 1992)

Los puntos de aforo fueron dispuestos siguiendo un orden jerárquico de cuencas integradas en la red de drenaje. Esta metodología facilitó una información espacio-temporal del transporte de sedimento, indicativo de sus ritmos de pulsación y sus relaciones con las condiciones climáticas. Asimismo, permitió establecer comparaciones cuantitativas entre las tasas de exportación o de erosión proporcionadas por ambientes característicos de cada área, y especialmente sobre la importancia relativa de las cárcavas.

La distribución de puntos de aforo (Fig. 2), integrados en orden de jerarquía creciente, se dispuso inicialmente de la siguiente manera:

1. El Carot $(0,1706$ ha): Parcela de erosión emplazada en el punto de salida de la red de drenaje de una microcuenca elemental en cárcavas, monitorizada mediante un sistema de colectores con divisores para la medición de agua y sedimentos.

2. Ca L'Isard (131,7 ha): Cuenca con cárcavas, bosques, prados, terrazas y campos de cultivo abandonados. Monitorizada mediante una estación de aforo, situada a la salida de la cuenca en el canal principal de drenaje. La estación fue equipada 


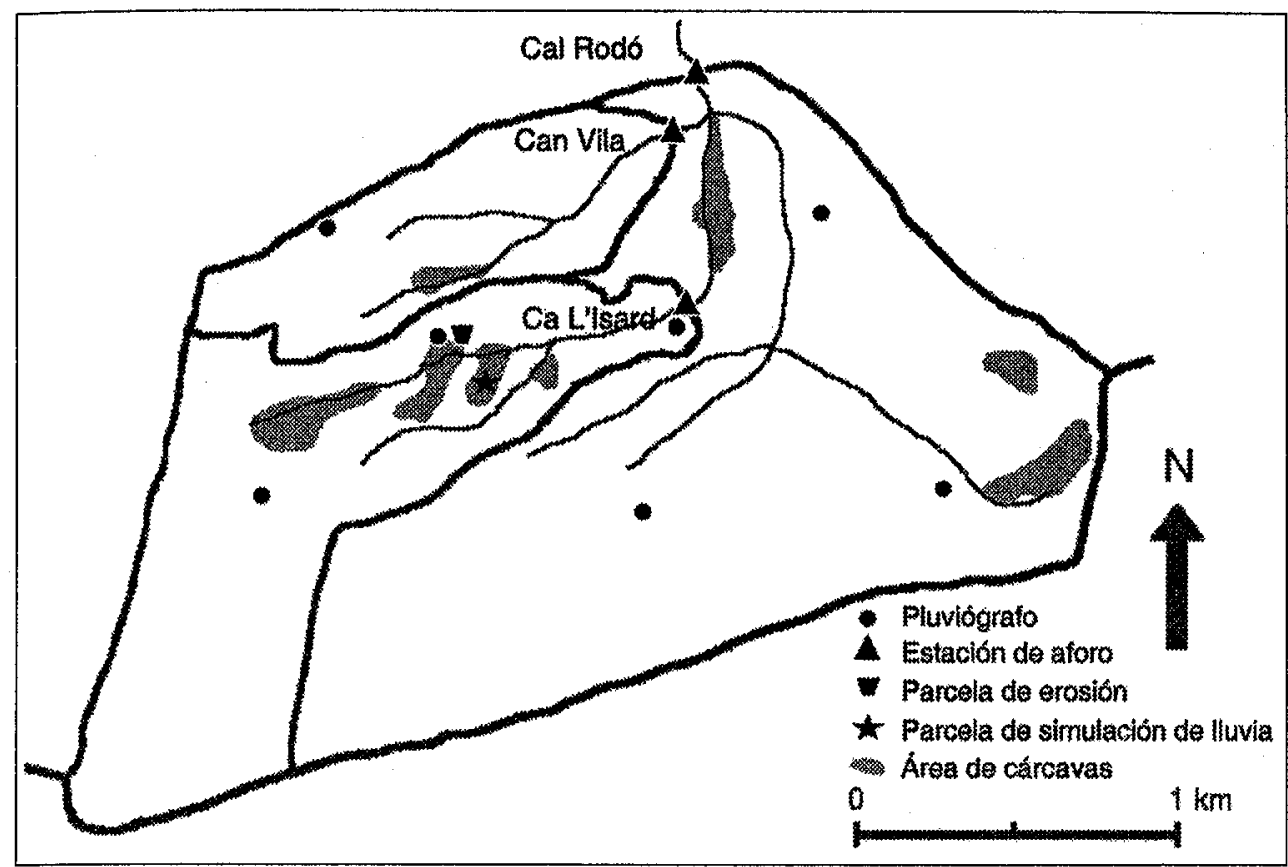

Figura 2. Distribución de las cuencas integradas, estaciones de aforo y pluviómetros en la cuenca de Cal Rodó mencionadas en el texto.

con un sensor de nivel, un sensor de turbidez de infrarrojos D\&A OBS-3, con un rango de medición de 0 a $5 \mathrm{~g} \mathrm{l}^{-1}$ y un muestreador automático (ISCO). Esta instrumentación proporcionó hidrogramas continuos, medidas puntuales de las concentraciones elevadas de sedimento durante las crecidas y sedigramas continuos en momentos de recesión y caudales de base.

3. Cal Rodó ( 417 ha): Cuenca con bosques, prados, terrazas y campos de cultivo abandonados, superficie estructural de calizas y cárcavas. En el punto de salida de la red de drenaje también fue instalada una estación de aforo con equipamiento similar a la de Ca L'Isard.

Los mencionados sensores de turbidez muestran un rango operativo insuficiente para la medición de concentraciones de sedimento durante las crecidas, además presentan dos inconvenientes principales: en primer lugar que requieren frecuentes calibraciones para garantizar un buen funcionamiento, y en segundo lugar que en momentos puntuales pueden proporcionar medidas erróneas por obturación de las sondas, como puede ocurrir por el crecimiento de algas, cubrimiento por hojarasca, ramas y sedimentos, formación de costras de calcio, etc., especialmente durante crecidas de gran magnitud. Por lo que es indispensable un mantenimiento frecuente y una buena selección de los puntos de instalación.

Esta necesidad queda reforzada si tenemos en cuenta que estos sensores ópticos miden la opacidad del agua, que está más relacionada con el número total de partículas en suspensión que con su masa, por lo que su respuesta depende de la composición mineral y el tamaño de las partículas transportadas (Gippel, 1995).

Asimismo, en las estaciones de aforo se cuantificó el transporte de solutos a partir de la medición de la conductividad eléctrica. Por otro lado, las precipitaciones 
se registraron mediante dos pluviómetros de balancín instalados junto a las estaciones de Ca L'Isard y la microcuenca de El Carot.

Resultados. El análisis de sedigramas e hidrogramas confirmó las hipótesis y observaciones referidas al control que ejerce la dinámica de las cárcavas sobre el transporte del sedimento (Clotet et al., 1988; Regüiés et al. 1995).

En primer lugar, la información recogida en la microcuenca de "El Carot" evidenció el efecto de la dinámica estacional de las cárcavas sobre las tasas de incorporación de sedimento a las redes de drenaje (Regüés, 1995; Castelltort, 1995; Balasch, 1998). Por otro lado, se observó que el transporte en las vertientes no es proporcional a las cantidades precipitadas, sino que está regulado por la distribución temporal y características de las lluvias.

Otra observación de interés se refiere a la modalidad de transporte, así el sedimento proporcionado por las cárcavas se transporta mayoritariamente en suspensión, representando más del $90 \%$, mientras que la carga de fondo apenas alcanza el $1 \%$ y el transporte en disolución constituye el segundo mecanismo en importancia (Castelltort 1995).

Con respecto a la variación espacio-temporal del "stock" de sedimento en cárcavas y canales de drenaje, se observó una buena correlación entre las observaciones en parcelas (Regües, 1995; Regüés y Gallart, 1996 y en prensa) y la evolución temporal de los sedimentogramas obtenidos en las crecidas registradas en Ca L'Isard y Cal Rodó. Así, entre primavera y principios del verano predomina la producción de sedimentos en las vertientes, mientras el transporte se produce mayoritariamente entre verano y otoño, coincidiendo con los periodos de máxima generación de escorrentía.

En cuanto a la cuantificación del transporte de sedimento, se obtuvo un primer balance del que derivaron las tasas de exportación anuales durante el periodo 199193 (Castelltort, 1995). En las cárcavas de "El Carot" se obtuvo una tasa media de $14.500 \mathrm{Mg} \mathrm{km}^{2} \mathrm{a}^{-1}$. Mientras en los puntos de aforo de Ca L'Isard y Cal Rodó las tasas medias indicaron valores de exportación inferiores en casi un orden de magnitud, 500 y $210 \mathrm{Mg} \mathrm{km}^{2} \mathrm{a}^{-1}$ respectivamente.

Estas diferencias parecen acordes con las variaciones de escala de observación, sin embargo la cuantificación en términos absolutos reveló diferencias significativas. En 1993 se observó un déficit de unos $400 \mathrm{Mg}$ entre el "stock" de sedimento estimado en las cárcavas de El Carot y el exportado de la cuenca de Ca L'Isard, este desajuste fue atribuido al comportamiento pulsante de los mecanismos de transporte. Además, esta diferencia sugirió que los años de mayor producción no siempre coinciden con los de mayor transporte y viceversa.

\section{Análisis espacio-temporal del transporte en las Cuencas integradas}

\subsection{Modificaciones introducidas en el diseño experimental}

Como hemos observado, la dificultad que conlleva el estudio y parametrización de algunos procesos geomorfológicos radica en su relación directa con causas cli- 
máticas, cuya variabilidad espacio-temporal genera un mosaico de posibilidades. Este problema puede ser amortiguado mediante la disponibilidad de un banco de datos de la mayor amplitud y calidad posible, que refleje el mayor número de matices relativos al proceso estudiado y por tanto permita establecer un elevado número de relaciones estadísticas de dependencia e independencia entre las variables aplicadas. Por otro lado, la correcta interpretación de la estadística esta supeditada a un conocimiento adquirido mediante la observación, las descripciones cualitativas y semicuantitativas, que permiten establecer aquellas relaciones no definidas por vía de la éstadística.

Con tal finalidad se implementaron las estaciones de Ca L'Isard y Cal Rodó mediante sistemas mejorados para la medición continua de elevadas concentraciones de sedimento. Esto permitiría evaluar el transporte cuando los muestreadores automáticos se revelan poco efectivos, como suele suceder en situaciones de: recesión de crecidas, caudales de base, crecidas demasiado rápidas y pequeñas o muy prolongadas, múltiples o encadenadas en breves intervalos de tiempo.

Para subsanar dicho problema fueron instalados dos sensores para la medición de sedimentos en suspensión (MOBREY msm40) basados en la atenuación de ultrasonidos (noviembre de 1994 en Ca L'Isard y junio de 1996 en Cal Rodó) lo que amplió el rango de medición del transporte en suspensión hasta unos $200 \mathrm{~g} \mathrm{l}^{-1}$.

Por otro lado, como complemento se amplió la red de pluviómetros, hasta un total de 7 puntos de captación de datos en toda la superficie de Cal Rodó (Fig. 2) (Latron y Gallart, 2001).

Para finalizar, el diseño de cuencas integradas quedó completado en 1995 , mediante la incorporación de la estación de aforo de Can Vila (Fig. 2), que proporcionó las tasas de exportación de sedimento en una cuenca sin cárcavas y con un importante cubrimiento vegetal. El punto de aforo fue equipado mediante un sensor de nivel, un muestreador automático ISCO (mayo de 1996) y un sensor de infrarrojos (julio de 1998).

\subsection{Mecanismos de transporte}

La Fig. 3a muestra las curvas de histéresis de caudales y sedimento de 6 crecidas correlativas registradas entre junio y septiembre de 1995 en Ca L'Isard, e ilustra un ejemplo típico de la evolución del transporte del sedimento durante el período de máxima disponibilidad. La principal característica observada es una disminución progresiva de la relación entre concentración de sedimentos y caudal. Las causas de este comportamiento se deben en primer lugar a un efecto de dilución (disminuye la concentración pero no el transporte), y después se va produciendo un cierto agotamiento del "stock" de sedimento disponible.

Esto queda evidenciado en la Fig. 3b, donde se observa como las cantidades de transporte no guardan una relación constante respecto a las cantidades de precipitación o su energía. Entre finales de primavera y principios de otoño, coincidiendo con una gran disponibilidad de sedimentos y el encostramiento del regolito superficial en las cárcavas, se producen las precipitaciones con mayor energía y eficacia erosiva en las vertientes. 

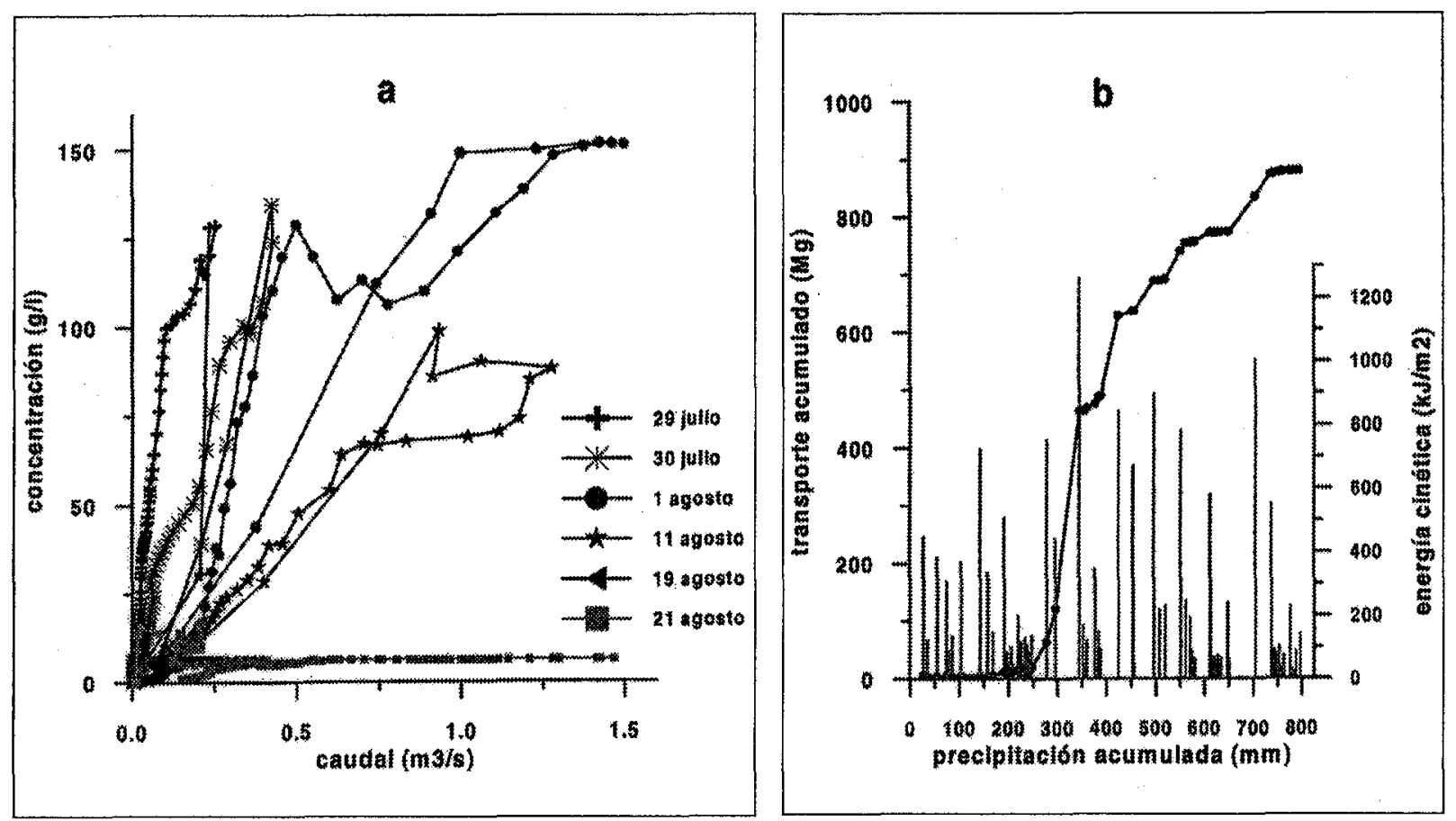

Figura 3. Ejemplo de la disminución temporal de la carga sólida específica cuantificada durante 1995 en la estación de Ca L'Isard. (a) Curvas de bistéresis de concentraciones de sedimento y caudales en 6 crecidas correlativas de gran magnitud.

(b) Secuencia anual de la producción de sedimento con respecto a las precipitaciones.

Posteriormente durante el otoño no se movilizan cantidades de material elevadas, teniendo en cuenta los importantes volúmenes de escorrentía registrados, pero esto no debe ser atribuido totalmente a un agotamiento del sedimento disponible, si no que es también una consecuencia de una gran producción de agua en las zonas vegetadas. Finalmente, durante el invierno y principios de primavera no se observa apenas transporte, como consecuencia de unos bajísimos coeficientes de escorrentía.

Las curvas de histéresis de caudales y concentraciones de sedimento (Fig. 4) indican dos mecanismos estacionales dominantes de transporte en suspensión (Castelltort, 1995; Gallart et al., 1998):

1. Crecidas en las que el pico de sedimento se produce antes que el pico máximo de caudal. Es lo que se denomina efecto "bulldozer". En estos casos el suministro de agua proviene del conjunto de la cuenca, mientras que los sedimentos proceden básicamente del pie de las vertientes y el lecho de los canales de drenaje principales. Esta modalidad está asociada a incrementos bruscos de la carga sólida al aumentar el caudal, lo que se debe a sucesivas roturas del cauce cada vez que la fuerza de arrastre del caudal supera la resistencia de cizalla que presentan los sedimentos, por lo que cada evento suele mostrar episodios correlativos de dilución y agotamiento. Este tipo de respuesta suele observarse desde principios o mediados del verano hasta el otoño; en este caso el encostramiento del regolito induce a la generación de flujos de escorrentía semi-Hortonianos, dado que la capacidad de saturación está limitada a unos pocos milímetros como consecuen- 
cia del encostramiento superficial, que facilita un sellado muy rápido y eficaz, lo cual justifica que las crecidas se produzcan de manera rápida y violenta, arrastrando fácilmente el sedimento disponible. En este caso la menor disponibilidad de sedimento se ve reflejada en concentraciones muy inferiores; respecto a las crecidas con características de histéresis antihoraria la proporción llega a ser de 50:1 (Fig. 4a), por ello solamente se producen concentraciones elevadas en momentos muy puntuales.

Así, este tipo de respuesta puede estar relacionado con dos causas: una mayor producción de agua por las áreas vegetadas, con concentraciones de sólidos muy inferiores, y un efecto de agotamiento del sedimento disponible, o incapacidad del caudal para erosionar el cauce tras el barrido inicial de los abanicos y depósitos incoherentes situados en la zona del canal de drenaje.

2. Crecidas en las que el aumento del caudal antecede al de sedimento. Típicas entre finales de invierno y el final de primavera o primera mitad de verano, cuando la mayor parte de la energía proporcionada por las precipitaciones se invierte en el encostramiento del regolito superficial y la erosión en las laderas de las cárcavas. En este caso la escorrentía procede casi exclusivamente de las cárcavas donde está limitada por una elevada capacidad de saturación hidráulica, causando flujos no Hortonianos en los que el incremento del caudal es demasiado lento para causar un transporte rápido desde las vertientes hasta los canales de drenaje principales. Esto queda reflejado en el retardo del pico de las concentraciones con respecto al del caudal (Fig. 4b). En este caso, si las precipitaciones son moderadas causan muy poco transporte con concentraciones bastante elevadas, por lo que la mayoría del sedimento movilizado es redepositado en la zona del cauce, pero cuando se producen tormentas intensas las concentraciones pueden alcanzar los $200 \mathrm{~g} \mathrm{l}^{-1}$ y el transporte alcanza valores elevados, aunque durante las recesiones también se produce redeposición en la zona del cauce.

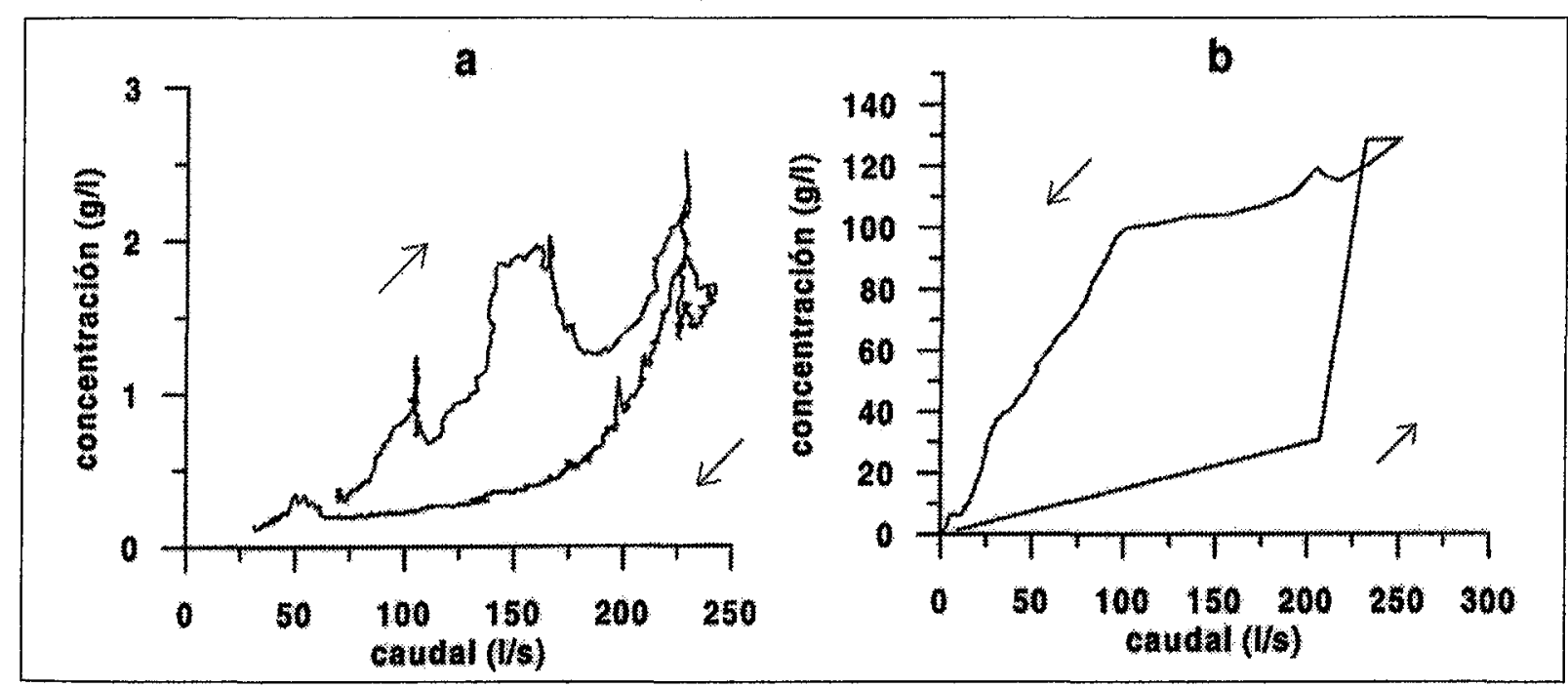

Figura 4. Curvas de histéresis observadas en la estación de Ca L'Isard: a) horaria, b) antihoraria. Obsérvese que las concentraciones en el ejemplo (a) son 50 veces inferiores a las expuestas en (b). 


\subsection{Resultados y discusión}

Los datos obtenidos, entre septiembre de 1994 y diciembre de 1999, procedentes de estaciones de aforo y pluviómetros, proporcionaron las medidas ininterrumpidas de caudales, concentraciones de sedimento y precipitaciones. Esta información complementada con el conocimiento de las condiciones físicas típicas estacionales en las cárcavas (Fig. 1) proporcionó un extenso volumen de datos para ser analizado.

El análisis de la información fue realizado mediante la aplicación de correlaciones lineales tomando tres escalas temporales, con la intención de establecer criterios objetivos de correlación entre el transporte y las características climático-estacionales. Esto, además de permitir un análisis estacional de los resultados, permitiría extrapolar las consecuencias hidrosedimentológicas que supondría un cambio de las condiciones climáticas o ambientales, dada la amplia variabilidad estacional registrada. De esta manera, las escalas de análisis seleccionadas fueron:

1. Escala de evento individual.

2. Escala mensual y estacional.

3. Escala anual.

Las variables independientes seleccionadas fueron: precipitación total, intensidad máxima de lluvia, energía cinética total y máxima, concentración de sedimentos media y máxima, caudal máximo, escorrentía total, coeficiente medio de escorrentía, día juliano, tiempo entre eventos, densidad aparente y humedad típicas antecedentes. Tomando como variable dependiente el transporte en suspensión.

El número total de crecidas registradas en cinco años y cuatro meses, fue de 367 en el caso de Ca L'Isard y 341 en el caso de Cal Rodó. Sin embargo, por problemas técnicos sucedidos en Cal Rodó, durante 1994 no se midieron correctamente las concentraciones de sedimento, por lo que dicho periodo no ha sido considerado en el análisis estadístico, aunque algunos gráficos y tablas reflejan los valores registrados. Así, el número de eventos analizados se redujo a 344 en Ca L'Isard y 300 en Cal Rodó.

Es importante indicar que los valores de precipitación utilizados corresponden únicamente a lluvias que causaron algún incremento del caudal y transporte de sedimento (mínimo $1 \mathrm{~kg}$ ) (tabla 1).

Tabla 1. Relación entre los registros anuales de precipitación total y las precipitaciones efectivas, en cuanto a incremento de caudal y transporte. Los valores con asterisco corresponden a una fracción del año.

\begin{tabular}{|c|c|c|c|c|}
\cline { 2 - 5 } \multicolumn{1}{c|}{} & $\begin{array}{c}\text { Precipitación total } \\
(\mathrm{mm})\end{array}$ & $\begin{array}{c}\text { Precipitación efectiva } \\
(\mathrm{mm})\end{array}$ & $\begin{array}{c}\text { (\%) de precipitación } \\
\text { considerado }\end{array}$ & $\begin{array}{c}\text { Int.máx.precipitación } \\
(\mathrm{mm} / \text { día })\end{array}$ \\
\hline $1994^{*}$ & $\mathbf{5 5 4}^{*}$ & $\mathbf{5 2 1 *}$ & $\mathbf{9 4 ^ { * }}$ & $\mathbf{1 1 8 ^ { * }}$ \\
\hline 1995 & $\mathbf{1 0 5 6}$ & $\mathbf{8 9 4}$ & $\mathbf{8 5}$ & $\mathbf{5 4}$ \\
\hline 1996 & $\mathbf{1 3 3 5}$ & $\mathbf{1 1 7 8}$ & $\mathbf{8 8}$ & $\mathbf{7 4}$ \\
\hline 1997 & $\mathbf{9 2 3}$ & $\mathbf{7 3 1}$ & $\mathbf{7 9}$ & $\mathbf{1 3 5}$ \\
\hline 1998 & $\mathbf{5 1 6}$ & $\mathbf{4 2 8}$ & $\mathbf{8 3}$ & $\mathbf{4 2}$ \\
\hline 1999 & $\mathbf{9 1 5}$ & $\mathbf{8 2 5}$ & $\mathbf{9 0}$ & $\mathbf{5 5}$ \\
\hline
\end{tabular}


Por otro lado también fueron estudiadas las 10 crecidas de mayor magnitud registradas en 1999 en la cuenca de Can Vila. En este caso no se aplicaron análisis estadísticos, a causa del reducido número de datos, insuficiente para estimar objetivamente los resultados. Por ello esta información solamente fue utilizada para comparar las tasas de transporte producidas entre áreas con cárcavas y vegetadas, aún presuponiendo que la tasa obtenida no debe experimentar grandes variaciones interanuales.

Escala de evento individual. La Fig. 5 muestra una comparación entre el porcentaje acumulado de precipitación y transporte producidos con respecto a los eventos acumulados. Observamos que aproximadamente un $90 \%$ del transporte total está relacionado con un $10 \%$ de las crecidas y un $25-30 \%$ de las precipitaciones.

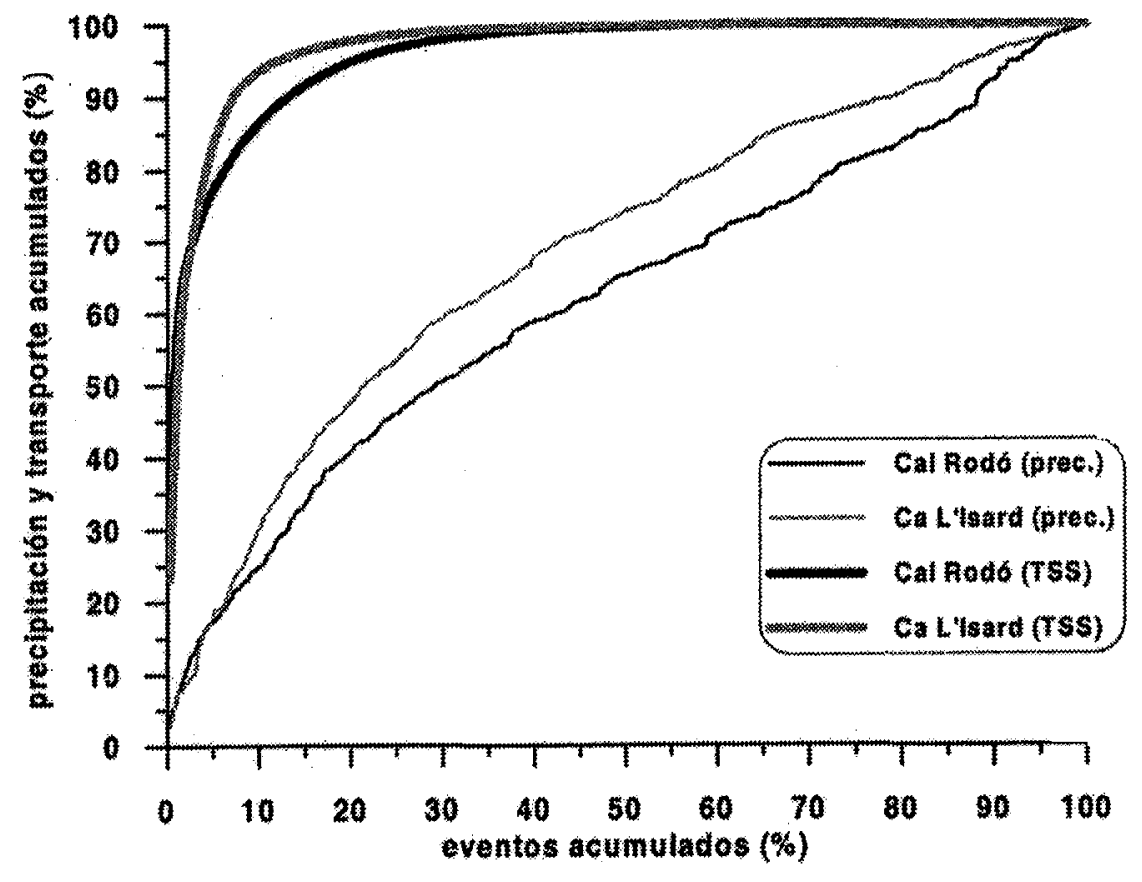

Figura 5. Precipitación y transporte de sedimentos acumulados en relación con el número de eventos.

En la tabla 2 están expuestos los resultados obtenidos de realizar regresiones múltiples entre los valores del transporte y las variables hidro-sedimentológicas registradas, además en dicho análisis se tomó en consideración la magnitud de las crecidas mediante una discriminación referida a las cantidades absolutas de transporte cuantificado, considerando cuatro umbrales: $1 \mathrm{~kg}$ (100\% del transporte total acumulado), $1 \mathrm{Mg}(99,75 \%), 10 \mathrm{Mg}(97,75 \%)$ y $100 \mathrm{Mg}(84,6 \%)$.

En relación con los resultados de los análisis estadísticos se tomaron dos intervalos de confianza para valorar los coeficientes de correlación $\mathrm{R}$, así se consideraron factores de peso aquellos que presentan un nivel de confianza mejor del $1 \%$, mientras las correlaciones con niveles de confianza entre el 1 y $5 \%$ fueron calificadas como débiles.

Una primera valoración de los resultados indica una relación directa entre número de variables correlacionadas y la cantidad de eventos considerados. Por otro lado, 
la comparación entre estaciones indica un número algo superior de variables correlacionadas en Ca L'Isard.

En general, los factores que mejor se correlacionan con el transporte en ambas cuencas son: la escorrentía total, el pico máximo de la crecida y la precipitación (energía cinética total); mientras algunas variables significativas al considerar las crecidas con baja capacidad de transporte pierden importancia tras discriminar los eventos de mayor entidad, este efecto se observa con las concentraciones, el coeficiente de escorrentía y el día Juliano.

También es importante señalar que los factores relacionados con el tiempo (día Juliano y tiempo entre crecidas) muestran correlaciones débiles únicamente con relación al día Juliano y en el caso de Ca L'Isard, además, dicha tendencia es independiente de la magnitud de los eventos.

El análisis conjunto de estos resultados es indicativo del desajuste entre los procesos de producción de sedimento y generación de escorrentía en las cárcavas (Regüés y Gallart, 1996 y en prensa). Así, tanto las pobres correlaciones obtenidas con respecto a la intensidad máxima de las precipitaciones, como su descenso progresivo al seleccionar los eventos de mayor magnitud, demuestran la influencia de la dinámica temporal de las condiciones físicas superficiales de las cárcavas sobre la respuesta hidrológica.

Tabla 2. Indices de correlación $R$ entre los factores relacionados con transporte de sedimentos y las variables consideradas a escala de evento individual. Los valores resaltados en negrita son estadísticamente significativos con un nivel de confianza mejor del $1 \%$ y en cursiva los que muestran un nivel mejor del $5 \%$.

\begin{tabular}{|c|c|c|c|c|c|c|c|c|}
\hline & \multicolumn{4}{|c|}{ Ca L'Isard } & \multicolumn{4}{|c|}{ Cal Rodó } \\
\hline & $\begin{array}{c}\text { Total Ts } \\
n=344\end{array}$ & $\begin{array}{c}\mathrm{Ts}>1 \mathrm{Mg} \\
\mathrm{n}=160\end{array}$ & $\begin{array}{c}\mathrm{Ts}>10 \mathrm{Mg} \\
\mathrm{n}=71\end{array}$ & $\begin{array}{c}\mathrm{Ts}>100 \mathrm{Mg} \\
\mathrm{n}=26\end{array}$ & $\begin{array}{c}\text { Total Ts } \\
n=300\end{array}$ & $\begin{array}{c}\mathrm{Ts}>1 \mathrm{Mg} \\
\mathrm{n}=180\end{array}$ & $\begin{array}{c}\mathrm{Ts}>10 \mathrm{Mg} \\
\mathrm{n}=94\end{array}$ & $\begin{array}{c}\mathrm{Ts}>100 \mathrm{Mg} \\
\mathrm{n}=18\end{array}$ \\
\hline Precipitación total. & 0,63 & 0,65 & 0,64 & 0,70 & 0,66 & 0,70 & 0,74 & 0,84 \\
\hline Ener. Cinética total & 0,60 & 0,61 & 0,60 & 0,64 & 0,63 & 0,67 & 0,70 & 0,81 \\
\hline Int.Precipitac máx. & 0,10 & 0,05 & $-0,06$ & $-0,03$ & 0,09 & 0,07 & 0,02 & 0,02 \\
\hline Ener.Cinética.máx. & 0,09 & 0,04 & $-0,06$ & $-0,03$ & 0,09 & 0,07 & 0,01 & 0,01 \\
\hline Escorrentia total & 0,78 & 0,77 & 0,77 & 0,74 & 0,70 & 0,70 & 0,71 & 0,71 \\
\hline Caudal máximo. & 0,64 & 0,64 & 0,60 & 0,57 & 0,64 & 0,65 & 0,65 & 0,65 \\
\hline Concentrac.móxima & 0,51 & 0,49 & 0,43 & 0,43 & 0,35 & 0,34 & 0,32 & 0,38 \\
\hline Concentrac.media & 0,45 & 0,42 & 0,33 & 0,07 & 0,20 & 0,16 & 0,11 & 0,17 \\
\hline Coefic.Escorrentia & 0,15 & 0,19 & 0,19 & 0,20 & 0,06 & 0,03 & 0,06 & 0,36 \\
\hline Tiempo entre Event. & 0,08 & 0,13 & 0,10 & 0,05 & $-0,01$ & 0,02 & 0,01 & 0,02 \\
\hline Día.Juliano & 0,12 & 0,15 & 0,23 & 0,37 & 0,07 & 0,09 & 0,11 & 0,16 \\
\hline Humedad Vol. $(b d)$ & 0,09 & 0,12 & 0,14 & 0,02 & 0,08 & 0,09 & 0,12 & 0,23 \\
\hline Densidad.Apar.(bd) & $-0,05$ & -0.09 & -0.11 & -0.01 & $-0,06$ & $-0,07$ & $-0,12$ & $-0,26$ \\
\hline
\end{tabular}

Esto no puede explicarse únicamente como un efecto de la conocida variabilidad espacial de la infiltración (Hawking, 1982; Fox et al. 1998) si no que debemos tomar en consideración que dicha variabilidad se ve afectada también por continuas 
variaciones temporales de propiedades que afectan directamente a la capacidad de infiltración, como son: conductividad hidráulica, capacidad de saturación, rugosidad hidráulica superficial o capacidad de retención hídrica, ligadas a cambios en la distribución y morfología de la porosidad (Pardini et al, 1996).

Estas particularidades son un ejemplo de la conocida irregularidad temporal y compulsividad de la erosión en situaciones de ambiente mediterráneo (Romero et al. 1986; Edwars and Owens 1991) causando en este caso una variedad de respuestas de características altamente no lineales, dado que el factor más determinante no depende únicamente de las características de las precipitaciones y la escasamente predecible distribución temporal de las tormentas, sino que está además afectada por la dinámica temporal de las propiedades hidráulicas y físicas que muestra el regolito en cada instante.

Este comportamiento no lineal explica que algunas variables de carácter semicuantitativo, como la intensidad máxima de precipitación, el espaciado temporal entre eventos o las condiciones físicas de las cárcavas, hayan mostrado coeficientes de correlación muy bajos al analizar individualmente los datos, dado que en estas condiciones las componentes cuantitativas son las que determinan un mayor peso al ser las únicas correlacionadas en grandes crecidas (Ts $>100 \mathrm{Mg}$ tabla 1). Asimismo, algunas de las relaciones analizadas requieren un ajuste mediante funciones no lineales para ser relacionadas con las características temporales del transporte, la confirmación de dicha hipótesis puede quedar de manifiesto mediante el análisis de los datos a escalas temporales menos detalladas.

Escala mensual y estacional. Los análisis estadísticos a escala mensual y estacional se realizaron a partir de los valores medios o máximos, dependiendo de la variable, mensuales y estacionales procedentes de la acumulación de datos en periodos anuales individuales o tomando el conjunto de 5 años. Mediante este procedimiento se obtuvo una información sintetizada en 60 y 12 periodos mensuales por un lado, y en 20 y 4 periodos estacionales. Esta simplificación permitió atenuar la gran dispersión de respuestas observada en los eventos individuales, otorgando mayor peso a las tendencias temporales directamente relacionadas con las condiciones climáticas, que a los fenómenos puntuales extraordinarios, poco relacionados en algún caso con las tendencias estacionales predefinidas. Además este procedimiento permite cierta capacidad de predicción de la respuesta que se podría esperar frente a una hipotética situación climática más cálida y árida, como sucede durante el verano.

La enorme influencia observada entre los eventos de gran magnitud y el transporte indicó la necesidad de observar el efecto de la distribución temporal de las tormentas, así fueron seleccionados tres rangos de precipitación diaria: 25, 50 y 100 $\mathrm{mm}$ y su presencia/ausencia en cada uno de los intervalos de tiempo considerados. a. Análisis mensual: El análisis estadístico mensual denotó el peso del número de casos en la significación estadística de las correlaciones (tabla 3). Así, el análisis del transporte considerando la acumulación de datos en 12 meses muestra un menor número de variables bien correlacionadas que con los 60 periodos mensuales. 
En general se observa que en el análisis de 60 meses existe una total coincidencia de las variables correlacionadas entre puntos de aforo, aunque el orden de importancia relativa entre ellas varía. Así en el caso de Ca L'Isard una la ordenación jerárquica de los factores correlacionados indican la influencia de la mayor proximidad de las cárcavas y el carácter pulsante del proceso de transporte, por dos motivos fundamentales:

1. En Ca L'Isard las tormentas de más de $50 \mathrm{~mm}$ muestran un mayor índice de correlación que las de más de $100 \mathrm{~mm}$, mientras en Cal Rodó se produce un incremento de los índices de correlación paralelamente al incremento de la magnitud de las precipitaciones.

2. Las concentraciones de sedimento muestran un peso muy superior en Ca L'Isard que en Cal Rodó.

Ambas observaciones indican que por Ca L'Isard el tránsito de sedimento es más regular y, por tanto, no depende tanto de la magnitud del caudal o del evento como en Cal Rodó, donde la exportación del material acumulado a lo largo del canal de drenaje requiere caudales de mayor entidad. En este caso, tal y como se observó en el análisis de eventos, los parámetros referentes a la distribución temporal, intensidad de precipitación y dinámica superficial en las zonas de cárcavas continúan denotando su independencia con respecto al transporte.

Tabla 3. Indices de correlación $R$ entre los valores de transporte de sedimentos (Ts) $y$ las variables sintetizadas a escala mensual de 5 años acumulados (12 casos) y considerados individualmente ( 60 casos). Los valores resaltados en negrita son significativos al 1 \% y en cursiva están indicados los que muestran un nivel de significación entre el 1 y $5 \%$.

\begin{tabular}{|l|c|c|c|c|}
\cline { 2 - 5 } \multicolumn{1}{c|}{} & Ca L'Isard $\mathrm{n}=12$ & Ca L'Isard $\mathrm{n}=60$ & Cal Rodó $\mathrm{n}=12$ & Cal Rodó $\mathrm{n}=60$ \\
\cline { 2 - 5 } \multicolumn{1}{c|}{} & Ts. & Ts. & Ts. & Ts. \\
\hline Mes & 0.66 & 0.25 & 0.57 & 0.22 \\
\hline$N^{\circ}$ de eventos & 0.30 & 0.11 & 0.28 & 0.07 \\
\hline Precipitación $(\mathrm{mm})$ & 0.53 & 0.41 & 0.59 & 0.43 \\
\hline Escorrentía $(\mathrm{mm})$ & 0.68 & 0.64 & 0.62 & 0.59 \\
\hline Int.máx.precipitación $(\mathrm{mm})$ & -0.20 & 0.03 & -0.12 & 0.04 \\
\hline Concentración máxima $\left(\mathrm{g} l^{1}\right)$ & 0.47 & 0.52 & 0.35 & 0.38 \\
\hline Concentración media $\left(\mathrm{g} l^{1}\right)$ & 0.62 & 0.57 & 0.39 & 0.34 \\
\hline Caudal máximo $\left(l \mathrm{~s}^{-1}\right)$ & 0.74 & 0.77 & 0.75 & 0.79 \\
\hline Coeficiente de escorrentia & 0.51 & 0.59 & 0.35 & 0.55 \\
\hline Humedad (0-10 cm) & 0.18 & 0.07 & 0.16 & 0.07 \\
\hline Densidad aparente $(0-5 \mathrm{~cm})$ & 0.05 & 0.02 & -0.01 & -0.01 \\
\hline Precipitación $>25 \mathrm{~mm}$ & 0.46 & 0.37 & 0.55 & 0.34 \\
\hline Precipitación $>50 \mathrm{~mm}$ & 0.73 & 0.64 & 0.64 & 0.57 \\
\hline Precipitación $>100 \mathrm{~mm}$ & 0.42 & 0.53 & 0.84 & 0.89 \\
\hline
\end{tabular}

En cuanto al análisis integrado en 12 meses confirma las observaciones anteriores, aunque en este caso se aumenta sensiblemente la correlación con respecto a la distribución temporal (significativo al 1,9 \% en Ca L'Isard y al 5,2 \%, en Cal Rodó). 
Esta observación resalta la existencia de fuertes diferencias interanuales, que enmascaran unas tendencias temporales que solamente se ponen de manifiesto mediante la agrupación de datos y que merecerían un estudio mediante análisis de varianza. Un aspecto que merece ser destacado es el descenso de significación de la precipitación total, especialmente evidenciado en el análisis de 5 años acumulados o simplificados en 12 periodos mensuales.

b. Análisis estacional. Los resultados obtenidos a partir del análisis estadístico estacional (tabla 4) indican que la acumulación de datos en 4 periodos estacionales causa una simplificación excesiva de la información y posiblemente suaviza las diferencias reales entre las tendencias características estacionales, además hay que tener en cuenta que la significación estadística de un análisis basado en una serie excesivamente corta de casos requiere coeficientes de correlación muy próximos a 1. Esto hace desaconsejable una simplificación excesiva de la información para este tipo de análisis.

En cuanto a los resultados obtenidos mediante el análisis de 20 periodos estacionales se observan ciertas diferencias entre ambos puntos de aforo, acordes con las observaciones y tendencias descritas anteriormente. En este caso queda claramente evidenciada la mayor importancia de la presencia/ausencia de precipitaciones de gran magnitud en detrimento de las cantidades totales precipitadas, mientras que una distribución estacional de los datos no indica diferencias temporales en el transporte apreciables estadísticamente. La proximidad de las cárcavas si queda reflejado en los resultados nuevamente, denotando que las características pulsantes del proceso prevalecen independientemente de la escala temporal de trabajo.

Tabla 4. Indices de correlación $R$ entre los valores de transporte de sedimentos (Ts) y las variables sintetizadas a escala estacional de 5 años acumulados ( 4 casos) y consideradas individualmente (20 casos). Los valores resaltados en negrita son significativos al $1 \% y$ en cursiva están indicados los que muestran un nivel de significación entre el 1 y $5 \%$.

\begin{tabular}{|c|c|c|c|c|}
\hline & Ca L'Isard $n=4$ & Ca L'Isard $n=20$ & Cal Rodó $n=4$ & Cal Rodó $n=20$ \\
\hline & Ts. & Ts. & Ts. & Ts. \\
\hline Estación climática & 0.88 & 0.40 & 0.83 & 0.35 \\
\hline$N^{o}$ de eventos & 0.13 & -0.05 & 0.35 & -0.06 \\
\hline Precipitación ( $m m$ ) & 0.38 & 0.24 & 0.53 & 0.27 \\
\hline Escorrentia $(\mathrm{mm})$ & 0.84 & 0.63 & 0.67 & 0.50 \\
\hline Int.máx.precipitación (mm) & -0.46 & -0.04 & -0.44 & -0.07 \\
\hline Concentración máxima $\left(g l^{\prime}\right)$ & 0.49 & 0.56 & 0.27 & 0.45 \\
\hline Concentración media $\left(g I^{I}\right)$ & 0.77 & 0.81 & 0.30 & 0.57 \\
\hline Caudal máximo $\left(l s^{-1}\right)$ & 0.08 & 0.79 & -0.03 & 0.77 \\
\hline Coeficiente de escorrentia & 0.25 & 0.63 & 0.00 & 0.42 \\
\hline Humedad $(0-10 \mathrm{~cm})$ & 0.10 & 0.06 & 0.03 & 0.02 \\
\hline Densidad aparente $(0-5 \mathrm{~cm})$ & 0.20 & 0.08 & 0.18 & 0.07 \\
\hline Precipitación $>25 \mathrm{~mm}$ & 0.29 & 0.19 & 0.41 & 0.12 \\
\hline Precipitación $>50 \mathrm{~mm}$ & 0.93 & 0.59 & 0.93 & 0.43 \\
\hline Precipitación $>100 \mathrm{~mm}$ & 0.58 & 0.45 & 0.77 & 0.87 \\
\hline
\end{tabular}


La Fig, 6 expone las distribuciones porcentuales de transporte, precipitaciones y tormentas por estación climática, lo que permite observar las relaciones entre ellos. En este caso se puede ver como no existe una relación de proporcionalidad entre cantidades de lluvia y transporte. Así, el transporte predomina durante el otoño, dónde un 25-30\% de las precipitaciones causa más del $55 \%$ del transporte, así como durante invierno el predominio de la meteorización queda reflejado en el mínimo anual de transporte, mientras el intervalo primavera-verano constituyen periodos de transición en los que las relaciones transporte/precipitación son muy inferiores a las observadas durante el otoño.

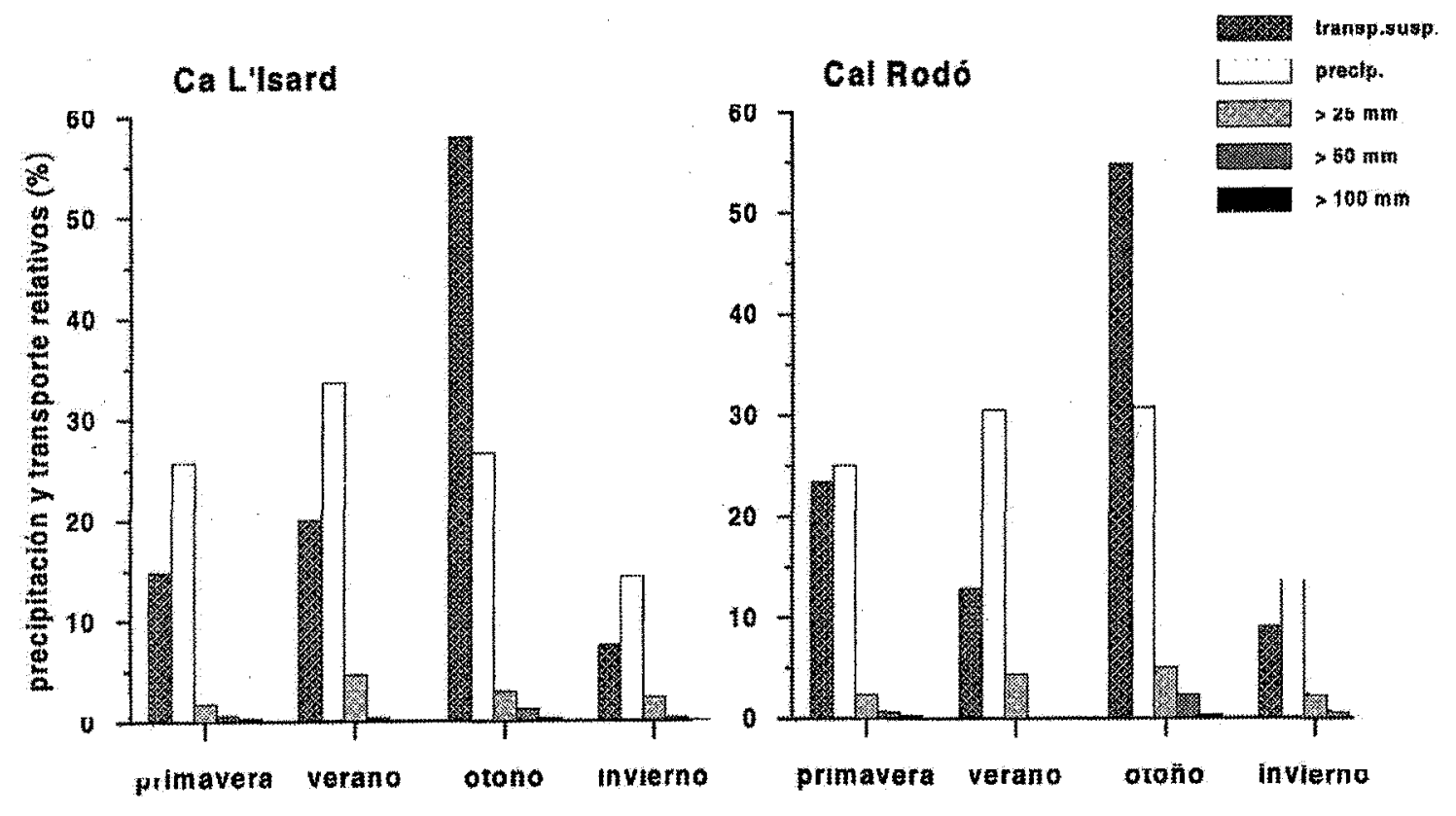

Figura 6. Porcentajes estacionales de transporte, precipitación y tormentas.

Por otro lado, la máxima distribución porcentual de las tormentas se produce durante el otoño, aunque aparecen en todas las estaciones climáticas pero su efecto, en cuanto al transporte, depende en gran parte de la disponibilidad de sedimento y la producción variable de escorrentía en las zonas de cárcavas.

Escala anual. Una observación de las tasas de transporte a escala anual (Fig. 7) muestra un elevado índice de variabilidad interanual, incluso de dos órdenes de magnitud en los casos más extremados. También es destacable observar como las relaciones transporte/pluviometría mantienen la misma tendencia observada a escala estacional, confirmando que la importancia de las precipitaciones no es solo cuantitativa sino que la situación temporal de las tormentas constituye el factor de mayor relevancia.

\subsection{Tasas de transporte de sedimentos}

Las tasas absolutas de transporte se han estimado para las cárcavas de El Carot a partir de los valores medidos en Ca L'Isard y Cal Rodó, asumiendo que la totalidad de los sedimentos proceden de las cárcavas. Como resultado se ha obtenido un 


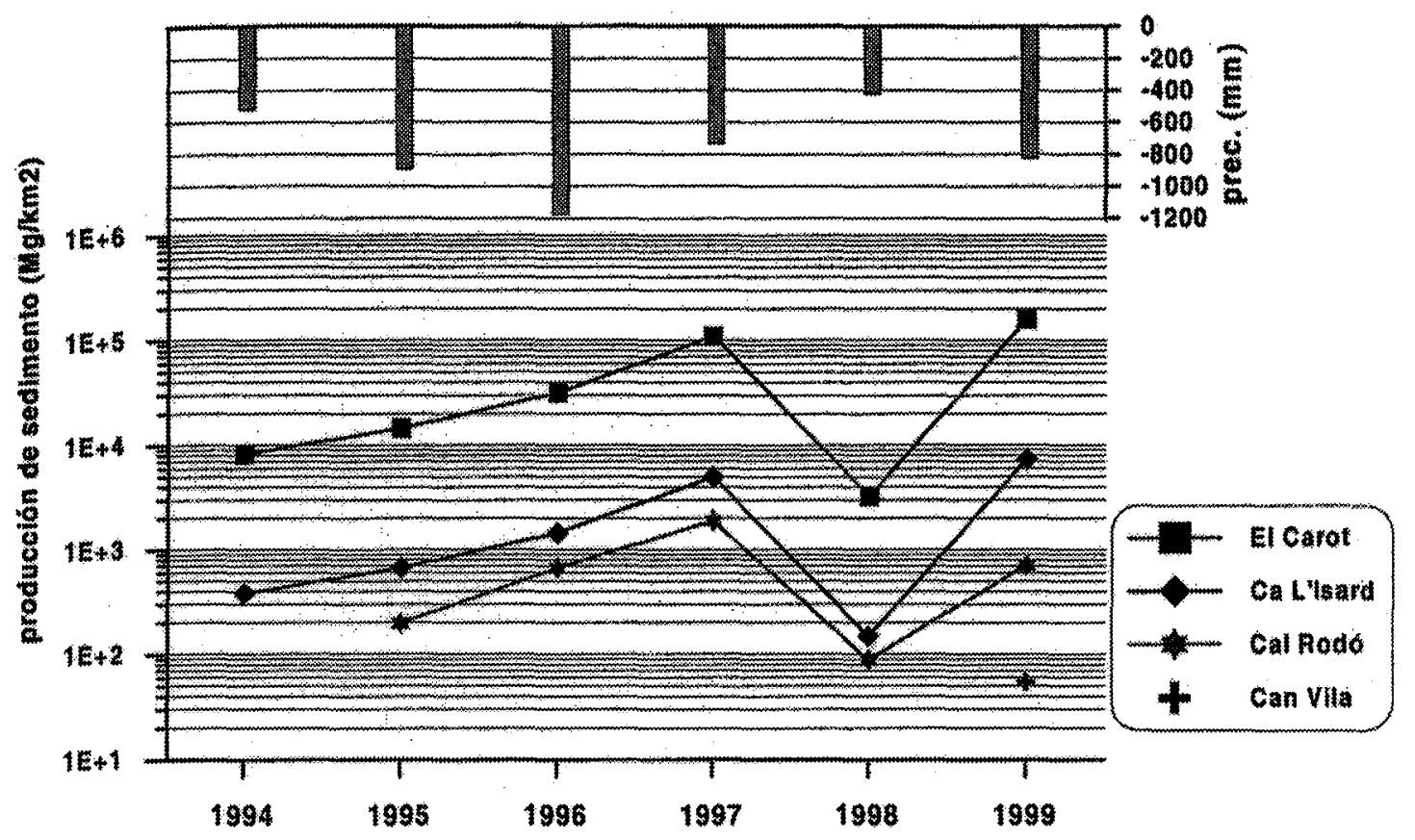

Figura 7. Tasas anuales de exportación de sedimento en suspensión y precipitación total.

valor de $60.000 \mathrm{Mg} \mathrm{km}^{-2} \mathrm{a}^{-1}$ que aún pudiendo parecer exagerado está en consonancia con los valores citados en multitud de trabajos. Así, la tasa máxima de transporte fluvial cuantificada a escala global es de unos $56.000 \mathrm{Mg} \mathrm{km}^{-2} \mathrm{a}^{-1}$, estimada por Walling y Webb (1996) en la cuenca de Huangfuchuan de $3.199 \mathrm{~km}^{-2}$ (río Yang-Tse, China), aunque las diferencias de escala hacen imposible una comparación objetiva, es evidente que en este caso los valores de erosión en las áreas fuente ha ser muy superior al registrado en El Carot.

Por otro lado Brochot (1993) obtiene unas tasas medias de erosión de unos $16.000 \mathrm{Mg} \mathrm{km}^{-2} \mathrm{a}^{-1}$ en las cárcavas de Roubine $\left(0,0013 \mathrm{~km}^{2}\right)$, situadas en una zona de montaña con una influencia climática mediterránea similar a la observada en $\mathrm{El}$ Carot, pero constituidas sobre materiales mucho más coherentes, en este caso las diferencia de escala no parecen ser tan determinantes como la propia susceptibilidad a la erosión de los materiales. Por otro lado, en algunos ríos de la cuenca Mediterránea se han calculado tasas de transporte superiores a $\operatorname{los} 4.000 \mathrm{Mg} \mathrm{km}^{-2} \mathrm{a}^{-1}$ (Webb et al., 1995) lo que implica la existencia de áreas fuente donde se producen tasas de erosión muy superiores a dicho valor.

Estas consideraciones de carácter general permiten analizar desde un punto de vista local la tasa de erosión media anual estimadas para las cárcavas de El Carot.y las cuantificadas en los 3 puntos de aforo:

Cárcavas en El Carot $\left(0,06 \mathrm{~km}^{2}\right): \quad 60.000 \quad \mathrm{Mg} \mathrm{km}^{-2} \mathrm{a}^{-1}$

Ca L'Isard $\left(1,317 \mathrm{~km}^{2}\right)$ :

2.800

Cal Rodó $\left(4,17 \mathrm{~km}^{2}\right)$ :

Can Vila $\left(0,56 \mathrm{~km}^{2}\right)::$ 
Como se puede observar el factor escala es determinante a la hora de efectuar la cuantificación de los datos, entre El Carot y Cal Rodó las tasas de erosión descienden dos órdenes de magnitud, aunque los valores obtenidos indican claramente que la producción de sedimentos está casi completamente asociada a zonas de cárcavas de muy reducida extensión, con respecto a zonas vegetadas (Can Vila) las diferencias relativas son de tres órdenes de magnitud.

Otro aspecto digno de mención es la diferencia entre la tasa media de erosión calculadas por Castelltort (1995) en 1991-93 y la estimada en 1995-99 con una instrumentación más sofisticada para el área de cárcavas de El Carot. Para el período 1991-93 se obtuvo un valor de $14.500 \mathrm{Mg} \mathrm{km}^{2} \mathrm{a}^{-1}$ mediante la información proporcionada por la parcela de erosión $\left(0,0017 \mathrm{~km}^{2}\right)$ mientras para los años posteriores se estima un valor 4 veces superior a partir de una información integrada del conjunto de la cuenca en que se encuentran las cárcavas, observándose en este caso unas variaciones interanuales muy elevadas (Fig. 7). Esto es indicador de que el grado de error que puede proporcionar la evaluación de tasas de erosión no está únicamente subyugado a la variabilidad temporal sino también a la disponibilidad de una instrumentación adecuada y a la situación del punto de medición.

Estas observaciones implican que los valores propuestos en este trabajo deben ser considerados con un razonable margen de credibilidad, pero no pueden tomarse como definitivos. Otro ejemplo de los problemas que comporta la medición de tasas de transporte se manifiesta en los valores calculados para Ca L'Isard y Cal Rodó, pues los valores estimados para la primera cuenca muestran una producción de sedimentos en términos absolutos de $3.700 \mathrm{Mg} \cdot \mathrm{a}^{-1}$ mientras para la segunda sería de unos 2.900 Mg. $\mathrm{a}^{-1}$, cuando Ca l'Isard está contenida en Cal Rodó.

\section{5. Transporte de solutos}

El transporte de sólidos disueltos viene fuertemente condicionado en Vallcebre por la abundante presencia de yesos, de modo que las concentraciones típicas son ligeramente superiores a $1 \mathrm{~g} \mathrm{l}^{-1}$. En la Fig. 8 se han representado las concentraciones de sólidos disueltos en las tres estaciones de aforo, en relación con los caudales líquidos. Puede observarse en esta figura el gran papel que juega el efecto de dilución, de modo que durante las crecidas, las concentraciones descienden más de medio orden de magnitud. Este descenso de la concentración no llega a compensar el efecto del caudal, de modo que el transporte total crece con el caudal. Una parte importante de la dispersión viene condicionada por la temperatura del agua, ya que ésta afecta fuertemente la solubilidad del sulfato de calcio.

Las concentraciones de solutos en las aguas de Can Vila son notablemente más bajas que en las de las otras cuencas a igual caudal, lo que en principio podría interpretarse como la consecuencia de la menor extensión de las zonas de cárcavas en esta subcuenca. Sin embargo, si tenemos en cuenta que el coeficiente de escorrentía medio anual es mayor en Can Vila, la menor concentración se puede atribuir a un mayor efecto de dilución. En efecto, las tasas anuales medias de disolución son de aproximadamente $600 \mathrm{Mg} \cdot \mathrm{km}^{-2}$ para Can Vila, $520 \mathrm{Mg} \cdot \mathrm{km}^{-2}$ para Ca l'Isard, y de $370 \mathrm{Mg} \mathrm{km}^{-2}$ para Cal Rodó. Si comparamos las pendientes de la figura 8, podemos 


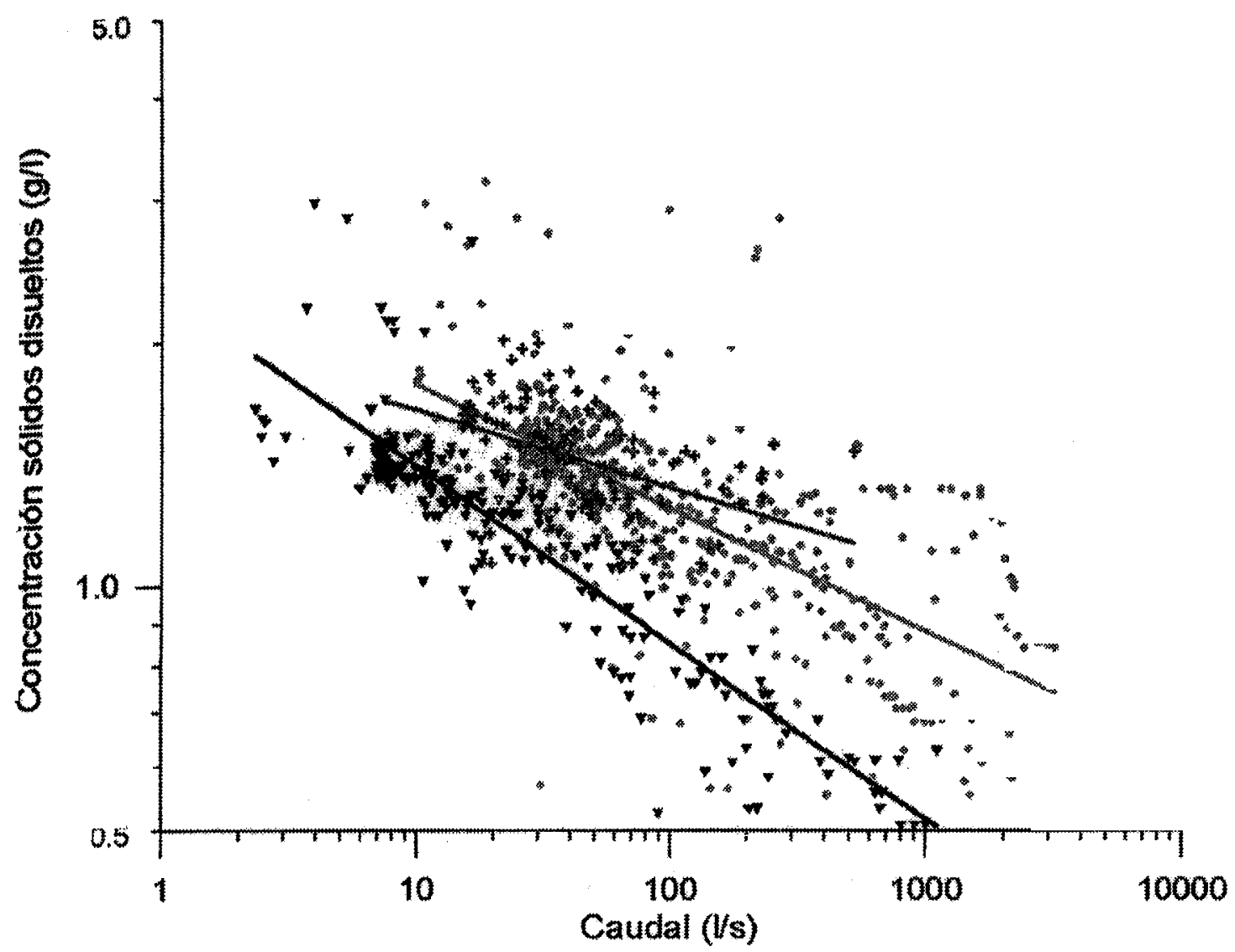

Figura 8. Concentraciones de sólidos disueltos en relación con el caudal en las estaciones de Can Vila (triángulos negros), Ca l'Isard (cruces, gris oscuro) y Cal Rodó (círculos, gris claro).

ver que la recta de Ca l'Isard tiene menor pendiente que las otras dos; esto puede interpretarse por el hecho de que los abundantes afloramientos de sulfato en las cárcavas en Ca l'Isard, favorecen que las aguas de arroyada se enriquezcan en sulfato durante las crecidas. De hecho. si comparamos las concentraciones de solutos con los valores de lámina de escorrentía en lugar del caudal, para compensar el tamaño de cuenca (gráfico no mostrado), las concentraciones en Can Vila aparecen prácticamente superpuestas a las de Cal Rodó, pero las de Ca l'Isard muestran un descenso menor con la escorrentía que en los otros dos casos.

\section{Conclusiones}

La complejidad observada en el estudio de los procesos de transporte en suspensión ha sido ilustrada por diversos autores: Walling $(1974,1978,1995)$, Carling (1983), Romero et al. (1986) o Edwars \& Owens (1991) entre otros. Coincidiendo todos ellos en la dificultad que entraña la parametrización y modelización de dicho proceso, originada en parte por la falta de información referente al transporte en cauces torrenciales o semitorrenciales y de transporte en vertientes (Govers 1992). Tal vez este constituye el aspecto más relevante en cuanto a justificar el interés asociado a la obtención de largas series de datos, con la esperanza de conseguir en el 
futuro modelos predictivos de erosión eficaces, lo cual se antoja imposible sin el soporte de una información extensa a niveles espacial y temporal.

Una consideración importante derivada de estos resultados, sería indicar la importancia de realizar estudios sobre transporte a medio-largo plazo. Una extrapolación de datos anuales a períodos más prolongados puede inducir a cometer errores que alcanzan en algún caso dos órdenes de magnitud, precisamente por la enorme compulsividad e impredivilidad con que se producen los procesos de erosión y transporte en éstas condiciones ambientales.

Con respecto a las consecuencias de un eventual calentamiento del clima sobre las zonas de cárcavas objeto de este estudio, parece que un aumento de las temperaturas podría asociarse a una disminución de las tasas de meteorización (Regües et al., 2000 ) lo que comportaría una menor disponibilidad de sedimento erodible. Sin embargo las condiciones climáticas características de las estaciones más cálidas (primavera-verano) son más favorables a la formación del encostramiento superficial, lo que facilita la generación de escorrentía y por tanto una respuesta hidrológica más rápida. Por tanto esta línea de razonamiento indica que un eventual incremento de temperaturas sin una alteración del régimen pluviométrico estacional propio de la zona presupondría posiblemente unas condiciones del regolito superficial menos variables, en las que una situación de encostramiento más persistente aumentaría el riesgo de una mayor generación de escorrentía, pero seguramente las tasas de erosión no serían tan elevadas como las cuantificadas en la actualidad.

Desde un punto de vista más integrado, hay que considerar que la existencia misma de badlands en Vallcebre ha sido interpretada como el resultado de la acción de la helada sobre el regolito y la vegetación (Regüés et al., 2000), de manera que un incremento de las temperaturas favorecería la revegetación espontánea de las cárcavas y su reducción o eliminación, en el mejor de los casos.

\section{Agradecimientos}

Este trabajo ha sido realizado mediante la financiación de los proyectos EPROHIDRO (HID98-1056-C02-01) y PROGESED (HID97-0581), y el acuerdo CSIC-DGCONA (RESEL). Los autores agradecen a Oscar Avila y Jérôme Latron su importante participación en los trabajos de diseño, instalación, puesta a punto y mantenimiento de las estaciones.

\section{Bibliografia}

BALASCH, J.C., 1986. La conca de Vallcebre (Alt Llobregat). Anàlisi de la dinàmica $g$ morfològica i balanç previ de sediments. Gr. Thesis. Facultad de Geología UB (Barcelona), $154 \mathrm{pp}$.

BALASCH, J.C., CASTELLTORT, X., LLORENS, P. \& GALLART, F., 1992. Hydrological and sediment dynamics network design in a Mediterranean mountainous area subject to gully erosion. In: Erosion and sediment transport monitoring programmes in river basins. IAHS Publ.210, pp.433.442. J.Bogen, D.E.Walling \& T.J.Day Eds. 
BALASCH, J.C., 1998. Resposta bidrològica i sedimentària d'una petita conca de muntanya analitzades a diferent escala temporal. PhD Thesis. Facultad de Geología UB (Barcelona), $376 \mathrm{pp}$.

BROCHOT, S., 1993. Modelisation du comportement hydrologique et erosif des bassins versants experimentaux de Draix (Alpes de Haute-Provence). In: Erosion de badlands dans le systeme Durance-Etang de Berre. 1êre partie. pp. 32 CEMAGREF Eds. Grenoble.

CARLING, P., 1983. Particulate dynamics, dissolved and total load, in two small basins, northern Pennines, UK Hydrol. Sci.J. 28, 355-375.

CASTELLTORT, X., 1995. Erosió, transport i sedimentació fluvial com a integració dels processos geomorfològics d'una conca. (Conca de Cal Rodó, Alt Llobregat). PhD Thesis. Facultad de Geología UB (Barcelona), 234 pp.

CLOTET, N. \& GALLART, F., 1986. Sediment yield in a mountainous basin under high mediterranean climate. Zeitschrift für Geomophologie, Sup. Bd., 60, 205-216.

CLOTET, N., GALLART, F. \& BALASCH, J.C., 1988. Medium term erosión rates in a small scarcely vegetated catchment in the Pyrenees. Catena supplement 13, Geomorphic processes, vol.II, 37-47.

EDWARDS, W.M. \& OWENS, L.B., 1991. Large storms effects on total soil erosion. Journ. Soil and Water Conservation, 46, 75-78.

FEIST, M. \& COLOMBO, F., 1983. Le limite Cretace-Tertiaire dans le EN de l'Espagne du point de vue des charofites. In: Colloquen Sénonien, Géologie Mediterranéenne. Tomo X (34), pp. 303-326.

FOX, D.S., BISSONAIS, Y. \& QUENTIN, P., 1992. The implications of spatial variability in surface hydraulic resistance for infiltration in a mound and depression microtopografphy. Catena, 32 (2), 101-114.

GALLART, F., LATRON, J. \& REGÜÉS, D., 1998. Hydrologycal and erosion processes in the research catchments of Vallcebre (Pyrenees). In: Modeling erosion by water. ANSI/NATO series 1-55, Berlín 487-498. Eds : J.Boardman \& D.Favis-Mortlock (SpringerVerlag).

GALLART, F., LATRON, J., LLORENS, P., SALVANY, M.C., JOSA, R., ANDERTON, S. \& WHITE, $S$. (in revision) Analysis of the hydrological functioning of a Mediterranean mountain catchment through monitoring of internal dynamics. Water Resources Research.

GIPPEL, CH.. J., 1995. Potential of turbidity monitoring for measuring the transport of suspended solids in streams. Hydrological Processes, 9, 83-97.

GOVERS, G., 1992. Evaluation of transport capacity formulae for overland flow. In: A.J. Parsons and A.D.Abrahams (Eds) Evaluation of transporting capacity formulae for overland flow. UCL, pp. 243-273.

GUÀRDIA, R., 1995. La colonització vegetal de les àrees erosionades de la conca de la Baells (Alt Llobregat). PhD Thesisl. Facultad de Biología UB (Barcelona), 317 p.

GUÀRDIA, R. \& NINOT, J.M., 1992. Distribution of the plant communities in the badlands of the upper Llobregat basin (southeastern Pyrenees). Studia Geobotanica, 12, 83-102.

HAWKINS, R.H., 1982. Interpretation of source-area variability in rainfall-runoff relationships. In: Rainfall-Runoff Relationships. Water Resource Publications.pp. 303-324. Eds: Singh V.P. For Collins Co. USA.

LATRON, J. \& GALLART, F., 2001. Spatial and temporal variability of rainfall in a small mediterranean mountainous catchment. In: Geophysical Research Abstracts. Hydrology and Earth System Sciences, vol. 3, pp. 374. 
LLORENS, P., QUERALT, I., PLANA, F. \& GALLART, F., 1997. Studying solute and particulate sediment transfer in a small mediterranean mountainous catchment subjet to land abandonment. Eartb Surface Processes and Landforms, 22, 1027-1035.

MARTÍNEZ, A. \& VERGÉS, J., 1978. Informe Geológico del Sector Fígols les Mines. Carbones de Berga S.A. Inédito.

MARTIN-VIDE, X., 1985. Estacionalidad de la precipitación y mediterraneidad en el Pirineo catalán. Notes de Geografía Física, 13-14, 57-65.

MCINTYRE, D.S., 1958. Soil splash and the formation of surface crust by raindrop impact. Soil Science, 85, 261-266)

PARDINI, G., 1996. Evoluzione temporale della microtopografia superficiale, della micromorfologia e della struttura in relazione ai processi di meteorizzazione nella marne smectiticbe di Vallcebre. PhD. Thesis. Facultad de Geología UB (Barcelona), 410 pp.

PARDINI, G., PINI, R., BARBINI, R., REGÜÉS, D., PLANA, F. \& GALLART, F., 1995. Performance of a laser profiling technique to evaluate the response of a smectite-rich mudrock subjected to thermic-misture cyclic changes. Soil Technology, 8, 161-175.

PARDINI, G., VIGNA GUIDI, G., PINI, R., REGÜÉS, D. \& GALLART, F., 1996. Structure and porosity of smectitic mudrock as affected by experimental wetting-driying cycles and frezzing-thawing cycles. Catena, 27, 149-165.

REGÜÉS, D., PARDINI, G., VIGNA GUIDI, G. \& GALLART, F., 1994. El efecto de los ciclos térmicos e hídricos sobre las rocas arcillosas, un experimento de laboratorio. In: Geomorfología en España. J.Arnáez, J.M. García Ruíz \& A. Gómez Villar (Eds.). Sociedad Española de Geomorfología. Logroño, pp. 63-72.

REGÜÉS, D., 1995. Meteorización física en relación con los procesos de producción y transporte de sedimentos en un área acarcavada. PhD. Thesis. Facultad de Geología UB (Barcelona), $302 \mathrm{pp}$.

REGÜÉS, D., PARDINI, G. \& GALLART, F., 1995. Regolith behaviour and physical weathering of clayey mudrock as dependent on seasonal wather conditions in a badland area at Vallcebre, Eastern Pyrenees. Catena, 25 (1-4), 199-212.

REGÜÉS, D. \& GALLART, F., 1996. Evolución de la respuesta hidrológica de un área arcillosa acarcavada, en relación con el estado físico superficial mediante experiencias de simulación de lluvia. Cadernos 21, 79-89.

REGÜÉS, D., GUÀRDIA, R. \& GALLART, F., 2000. Geomorphic agents versus vegetation spreading as causes of badland occurrence in a Mediterranean subhumid mountainous area. Catena. Special Issue: Badlands in changing environments, 40 (2), 173-187.

REGÜÉS, D. \& GALLART, F. (en prensa). Temporal patterns of runoff and erosion rates in a mountain badland area at Vallcebre basin (south-east Pyrenees). Earth Surface Processes and Landforms.

ROMERO, M.A., LÓPEZ-BERMÚDEZ, F., THORNES, J.B., FRANCIS, C. \& FISHER, G.C., 1988. Variability of overland flow erosion rates in a semiarid mediterranean environment under matorral cover, Murcia, Spain. Catena Sup., 13, 139-146.

SLATTERY, M.C. \& BRYAN, R.B., 1994. Surface seal development under simulate rainfall on an actively eroding surface. Catena, 22, 17-34.

SOlÉ, A., JOSA, R., PARDINI, G., ARINGHIERI, R., PLANA, F. \& GALLART, F., 1992. How mudrock and soil physical properties influence badland formation at Vallcebre (Pre-Pyrenees, EN Spain). Catena, vol. 19, (3-4), pp. 287-300. 
WALLING, D.E., 1974. Suspended sediment and solute yields from a small catchment prior to urbanisation. In: Fluvial Processes in Instrumented Watersheds, Institute of British Geographers Special Publication $\mathrm{n}^{\circ}$ 6, pp. 169-192.

WALLING, D.E., 1978. Suspended sediment and solute response characteristics of the River Exe, Devon, England. In: Research in Fluvial Geomorpbology (Proceedings of 5th Guelph Symposium on Geomorphology), Davidson-Arnott, R. and Nickling, W. (eds.), Geo Books, Norwich, pp. 169-197.

WALLING, D.E., 1995. Suspended sediment transport by rivers: a geomorphological and hydrological perspective. In: Proceedings of the International Symposium on Particulae Matter in Rivers and Estuaries (Reinbek/Hamburg).

WALLING, D.E. \& WEBB, B.W., 1996. Erosion and sediment yield: a global overview. In: Erosion and sediment yield: global and regional perspectives. LAHS Publ. 236, 3-19. D.E. Walling \& B.W. Webb (Eds.)

WEBB, B.W., FOSTER, I.D.L., GURNELL, A.M., 1995. Hydrology, Water Quality and Sediment Behaviour. In: Sediment and Water Quality in River Catchments. Ian Foster, Angela Gurnell \& Bruce Webb Eds. Ed: John Wiley \& Sons Ltd, pp. 1-30. 